\title{
Bifurcation, Chaos, and Voltage Collapse in Power Systems
}

\author{
CHIN-WOO TAN, MATTHEW VARGHESE, PRAVIN VARAIYA, FELLOW, IEEE, AND \\ FELIX F. WU, FELLOW, IEEE
}

\author{
Invited Paper
}

\begin{abstract}
A model of a power system with load dynamics is studied by investigating qualitative changes in its behavior as the reactive power demand at a load bus is increased. In addition to the saddle node bifurcation often associated with voltage collapse, the system exhibits sub-and supercritical Hopf bifurcations, cyclic fold bifurcation, and period doubling bifurcation. Cascades of period doubling bifurcations terminate in chaotic invariant sets. The presence of these new bifurcations motivates a reexamination of the saddle-node bifurcation as the boundary of the feasible set of power injections.
\end{abstract}

\section{INTRODUCTION}

Our objective is to study bifurcations in the behavior of a power system as a system parameter (e.g., load) is quasistatically varied. By definition, bifurcations occur at those parameter values where there is a change in the system's qualitative properties. Power systems are operated at a stable operating point and, under normal conditions, the operating point varies smoothly with changes in the system parameter, so the variation can be tracked by local, linear analysis. However, the linear analysis breaks down when bifurcations occur, and one must resort to more global, nonlinear analysis. Bifurcations also profoundly affect system behavior even under normal conditions, because they constrain the region of attraction of a stable operating point. Thus bifurcations influence both the range of stable operating points as well as the transient stability properties of those operating points. This is what makes the study of bifurcations useful from an engineering viewpoint.

We study static bifurcations, dynamic bifurcations and, especially, chaos $^{1}$ in power systems. A static bifurcation

Manuscript received June 18, 1994; revised August 9, 1995. This work was supported by the Electric Power Research Institute.

C. W. Tan is with GeoStar Technology Inc., Santa Clara, CA 95051 USA.

M. Varghese, P. Varaiya, and F. F. Wu are with the Electrical Engineering and Computer Sciences Department, University of California, Berkeley, CA 94720 USA.

IEEE Log Number 9414947.

${ }^{1}$ Chaos is a concept describing bounded, unstable behavior. More detailed definitions follow in Section III. implies a change in the number of equilibria. A dynamic bifurcation can be a Hopf, cyclic fold, or period doubling bifurcation. A Hopf bifurcation causes the emergence or disappearance of a periodic solution from an equilibrium point [25]. A cyclic fold bifurcation occurs when a stable limit cycle and an unstable limit cycle collide and disappear. A period doubling bifurcation refers to the emergence of a periodic orbit near another periodic orbit. Static and Hopf bifurcations are local phenomena. Chaos emerges from a global bifurcation. This means there is a qualitative change in system trajectories not restricted to a small neighborhood of an equilibrium point.

The classical (swing equation) model of a power system exhibits both local and global bifurcations. Static bifurcations are always present because the power flow equations have multiple solutions. In power systems where voltages are determined by reactive power flows, these bifurcations can shrink the region of stability. An electrical subsystem, such as the exciter, can interact with machine dynamics to produce oscillations via a subcritical Hopf bifurcation. This, too, shrinks the region of stability. As will be seen, the occurrence of chaos can similarly affect the behavior around an operating point.

The paper is organized as follows. In Section II, we review bifurcations that are relevant to our study. In Section III, we discuss chaos and related complicated dynamic behavior. Where appropriate, we draw examples from power systems. In Section IV, simulation results of a classical power system model augmented by a dynamic load are presented. Others have studied the same model [3], [5], [6]. The global asymptotic picture of even this simple system is incomplete, although local bifurcations near an equilibrium or a periodic orbits are well understood. Some concluding remarks are collected in Section V.

The classical models with positive damping and lossless transmission lines have relatively simple behavior. Because they possess energy functions that can also serve as 
Lyapunov functions, they have no limit cycles. ${ }^{2}$ Trajectories either converge to equilibrium points or are unbounded. The existence of unbounded trajectories depends on whether machine angles are measured $[0,2 \pi)$ or $(-\infty,+\infty)$. The same conclusion may be drawn for more general structure preserving systems (with damping), because they too possess energy functions. General algorithms for the construction of these functions are available in [19], [21], and [31]. For the classical and structure preserving models, saddle-node bifurcation (SNB) ${ }^{3}$ is the only possible local bifurcation. Analytical characterizations of the exact boundary of the stability region of a stable equilibrium point are available in [12], [35]. For some systems, this boundary has a "tangled" shape [15]. Details on how this affects the construction of Lyapunov functions are given in [33].

Global bifurcations can be direct consequences of local bifurcations. Unstable limit cycles contribute to this transition by forming homoclinic orbits with other limit sets. A list of such transitions is given below. More detailed definitions can be found in Sections II and III.

1) Cyclic Fold Bifurcation: This is an important bifurcation for the power system example considered in Section IV. An unstable limit cycle and a stable limit cycle are created. The shrinkage of the stability domain of the stable operating point commences with the appearance of a pair of unstable-stable limit cycles.

2) Period Doubling: The study in [4] for a pair of generators connected to a lossy line shows that in the case of a supercritical Hopf bifurcation, the stability of the periodic orbit is short-lived. When the stability of the periodic orbit is lost, another periodic orbit of twice its period becomes stable. If this pattern continues, it is known as period doubling.

3) Homoclinic Chaos: In the classical power system model with no damping, it is possible to obtain complicated trajectories provided that the machine angles are measured in $[0,2 \pi)$. Suppose a saddle connection $^{4}$ in the machine dynamics of a single swing equation in $[0,2 \pi) \times \Re$ is perturbed by a periodic solution from another part of the power system. Suppose the periodic forcing perturbation term breaks up the saddle connection so as to produce a homoclinic tangle. ${ }^{5}$ This global bifurcation results in chaos.

As mentioned, certain types of bifurcations cannot occur in the classical models. There is a question about validation of models which do exhibit those behaviors. In the last instance, validation should be based on observed data. However, observed data are collected over a finite time interval, whereas phenomena like chaos characterize behaviors over

\footnotetext{
${ }^{2}$ If the machine angles are measured in $[0,2 \pi)$, there may be limit cycles.

${ }^{3} \mathrm{SNB}$ occurs when a stable-unstable pair of equilibrium points collide and disappear.

${ }^{4} \mathrm{~A}$ saddle connection occurs when trajectories connect pairs of saddle equilibrium points.

${ }^{5} \mathrm{~A}$ homoclinic tangle is defined in Section III.
}

arbitrarily long time intervals. Thus the occurrence of these behaviors must be inferred indirectly. Often they are manifested as experimental or numerical anomalies. For example, some bifurcations can produce numerically and experimentally unobservable quantities such as unstable limit cycles and fractals like the Cantor set. ${ }^{6}$ Even if one were to accept these models as valid, it is usually not possible to prove the occurrence of chaos, and one has to resort as we do to interpretations of simulation results that lend support to the claim of occurrence of chaos. Thus much theoretical and experimental work remains to be done before one can accept that certain complicated behaviors are characteristic of power systems.

\section{REVIEW OF BIFURCATION TERMINOLOGY}

The mathematical literature on bifurcation theory is immensely rich and diverse. A large number of definitions and concepts have been developed to deal with the qualitative behavior of the orbit structure of parametrized families of autonomous dynamical systems. Static bifurcations were first related to voltage collapse in [23] and nonlinear oscillations in power systems were identified with Hopf bifurcations in [1], [4], and [27]. A companion paper in this volume also analyzes local bifurcations. An EPRI report provides a review of the literature [32]. In this section, we present the basic definitions of cyclic fold, period doubling and the formation of invariant tori. These phenomena can be viewed as local bifurcations on a suitably chosen Poincaré section.

\section{A. Cyclic Fold, Period Doubling, and Invariant Tori}

A cyclic fold bifurcation occurs when a stable limit cycle collides with an unstable one, and both disappear as the system parameter is further varied. A period doubling bifurcation refers to the emergence of a stable periodic orbit $\Gamma_{2}$ near another periodic orbit $\Gamma_{1}$, where the period of $\Gamma_{2}$ is approximately twice of that of $\Gamma_{1}$. The orbit $\Gamma_{1}$ is stable before the bifurcation, but loses its stability after the bifurcation. The bifurcation of a periodic orbit for a differential equation can be studied as a local bifurcations of a fixed point ${ }^{7}$ of the Poincaré map $\mathcal{P}$. We first review the idea of a Poincaré map for a periodic orbit.

Consider a differential equation $\dot{x}=f(x), x \in \Re^{n}$. Denote its solution starting at $x$ by $\phi_{t}(x)=\phi(t, x)$. The map $\phi:(t, x) \rightarrow \phi(t, x)$ is called the flow of the vector field $f(x)$. Let $\Gamma$ be a periodic orbit of period $T$ of the flow $\phi$. We first take a local cross section $S \subset \Re^{n}$, of dimensional $n-1$, such that 1 ) the flow $\phi$ is everywhere transverse $^{8}$ to $S$, and 2) $S$ intersects $\Gamma$ at a unique point

\footnotetext{
${ }^{6} \mathrm{~A}$ fractal is a set with a fractional dimension. The dimension of a set is defined to be $D$ if the following covering of the set is implemented. Suppose one were to cover the set with hypercubes of side $\epsilon$. Then, $N(\epsilon)$, the minimum number of such hypercubes needed, would scale as $N(\epsilon) \sim \epsilon^{-D}$ when $\epsilon \rightarrow 0$. For a line segment $D$ is 1 , for a plane $D$ is 2. For a Cantor set $D$ is an irrational number between 1 and 2 .

${ }^{7}$ A point $x_{0}$ is a fixed point of a map $x \mapsto f(x)$ if $f\left(x_{0}\right)=x_{0}$.

${ }^{8}$ This means the inner product $\langle f(x), n(x)>\neq 0$ for all $x \in S$, where $n(x)$ is the normal to $S$ at $x$.
} 
$p$. Let $\mathcal{U} \subset S$ be a neighborhood of $p$. For $q \in \mathcal{U}$ the first return or Poincaré map $\mathcal{P}: \mathcal{U} \rightarrow S$ is defined by

$$
\mathcal{P}(q):=\phi_{\tau(q)}(q)=\phi(\tau(q), q)
$$

where $\tau(q)$ is the time it takes for the orbit based at $q$ to first return to $S$. Thus $\tau(p)=T$ and $\tau(q) \rightarrow T$ as $q \rightarrow p$. Also $p$ is a fixed point of $\mathcal{P}$. In local coordinates, $\mathcal{P}$ is a map from $\mathcal{U} \subset \Re^{n-1}$ to $\Re^{n-1}$, so $D \mathcal{P}(p)$ has $n-1$ eigenvalues, called the Floquet multipliers (or characteristic multipliers) associated with the periodic orbit $\Gamma$.

Theorem 2.1: The periodic orbit $\Gamma$ is orbitally asymptotically stable if all the Floquet multipliers have magnitude less than one. Moreover, $p$ is a stable fixed point for the Poincaré map if $\Gamma$ is stable.

Consider a one-parameter family of differential equations $\dot{x}=f(x, \mu)$. Suppose it has a periodic orbit for a parameter value $\mu$. Denote the Floquet multipliers by $\lambda_{1}(\mu), \cdots, \lambda_{n-1}(\mu)$. If the periodic solution is stable, then by Theorem $2.1 \lambda_{1}(\mu), \cdots, \lambda_{n-1}(\mu)$ are all inside the unit circle. The multipliers are functions of the parameter $\mu$. As $\mu$ is varied, some of them may cross the unit circle at a critical value $\mu_{0}$. A multiplier crossing the unit circle is called a critical multiplier. Several types of bifurcation may occur depending on how a multiplier or a pair of complex conjugate multipliers crosses the unit circle. There are three typically possible cases:

1) Only one multiplier crosses the unit circle along the positive real axis with $\lambda_{i}\left(\mu_{0}\right)=1$ for some $i$.

2) Only one multiplier crosses the unit circle along the negative real axis with $\lambda_{i}\left(\mu_{0}\right)=-1$ for some $i$.

3) Only one pair of complex conjugate multipliers crosses the unit circle with $\left|\lambda_{i}\left(\mu_{0}\right)\right|=1$, $\operatorname{Im}\left(\lambda_{i}\left(\mu_{0}\right)\right)>0$, and $\frac{d}{d \mu}\left|\lambda_{i}\left(\mu_{0}\right)\right| \neq 0$ for some $i$.

If $p \in \Gamma, \Gamma$ a periodic orbit for a parameter $\mu$, we say that $p$ is a hyperbolic fixed point for the (appropriate) Poincaré map $\mathcal{P}_{\mu}$ if none of the multipliers $\lambda_{1}(\mu), \cdots, \lambda_{n-1}(\mu)$ lies on the unit circle. All the above three cases refer to a loss of orbital stability when $\mu$ approaches $\mu_{0}$ from the appropriate side (Conversely, the system gains stability if critical multipliers enter the unit circle). Thus there is a bifurcation at $\mu_{0}$. Exactly like in the case for differential equations, the lack of hyperbolicity causes bifurcations for a mapping. Without loss of generality, we assume $\lambda_{1}\left(\mu_{0}\right)$ is the critical multiplier in both case 1 and case 2 , and $\lambda_{1}\left(\mu_{0}\right)=\overline{\lambda_{2}}\left(\mu_{0}\right)$ is the conjugate pair of multipliers in case 3 . Proofs of the following results may be found in [8] and [34].

Case 1: [Cyclic fold] $\lambda_{1}\left(\mu_{0}\right)=1$ A fixed point for $\mathcal{P}_{\mu}$ is a point on a periodic orbit that solves the equation

$$
\mathcal{P}_{\mu}(q)-q=0, q \in S_{\mu} .
$$

Suppose $p_{0}$ is a fixed point for $\mathcal{P}_{\mu_{0}}$. Since $\lambda_{1}\left(\mu_{0}\right)=1$, $D_{q} \mathcal{P}_{\mu_{0}}\left(p_{0}\right)-I$ is not invertible, where $I$ is the $(n-1) \times(n-$ 1) identity matrix. So we cannot apply the implicit function theorem to get a smooth fixed point solution $p(\mu)$ with $p_{0}=$ $p\left(\mu_{0}\right)$. Indeed the fixed point solution undergoes a saddlenode bifurcation at $\mu_{0}$. This corresponds to a coalescence of two hyperbolic fixed points, one stable and the other unstable, as $\mu$ approaches $\mu_{0}$. The stable (respectively, unstable) fixed point lies on a stable (respectively, unstable) periodic orbit. Hence a cyclic fold bifurcation of periodic orbits corresponds to a (local) saddle-node bifurcation of the fixed point solution for the Poincaré map.

Cyclic fold bifurcation occurs for the power system example in Section IV.

Case 2: [Period doubling] $\lambda_{1}\left(\mu_{0}\right)=-1$ Suppose $p_{0}$ is a stable fixed point for $\mathcal{P}_{\mu_{0}}$. The implicit function theorem gives a smooth fixed point solution $\mu \rightarrow p(\mu)$ through $\left(p_{0}, \mu_{0}\right)$. However, the solution branch loses its stability and a stable periodic orbit consisting of two periodic points? of period 2 is present for either $\mu>\mu_{0}$ or $\mu<\mu_{0}$ depending on whether $\frac{d}{d \mu}\left|\lambda_{1}\left(\mu_{0}\right)\right|>0$ or $\frac{d}{d \mu}\left|\lambda_{1}\left(\mu_{0}\right)\right|<$ 0 . We say that the fixed point solution has a pitchfork bifurcation at $\mu_{0}$. Suppose $\mu$ near $\mu_{0}$ is a parameter after the bifurcation has occurred. Let $p_{1}(\mu), p_{2}(\mu)$ be the two period 2 periodic points for the Poincare map $\mathcal{P}_{\mu}$. Since $\mathcal{P}_{\mu}\left(p_{1}(\mu)\right)=p_{2}(\mu)$ and $\mathcal{P}_{\mu}\left(p_{2}(\mu)\right)=p_{1}(\mu)$, a new periodic orbit of approximately twice the period of the periodic orbit which contains $p(\mu)$ is created, and it intersects the Poincaré section at $p_{1}(\mu)$ and $p_{2}(\mu)$. Thus a period doubling bifurcation of a periodic orbit corresponds to a (local) pitchfork bifurcation of the fixed point solution for the Poincaré map.

The power system model in Section IV as well as an example involving two generators in [4] both undergo period doubling bifurcation.

As an example, consider the logistic map [26] defined by

$$
F_{\mu}(x):=\mu x(1-x), \quad 0 \leq x \leq 1, \quad 1 \leq \mu \leq 4 .
$$

For $1 \leq \mu \leq 4, F_{\mu}$ maps $[0,1]$ to $[0,1]$. Solving the equation $F_{\mu}(x)=x$ gives the fixed points 0 and $x^{*}(\mu)=$ $\frac{\mu-1}{\mu}$. For $x^{*}(\mu) \in[0,1]$, we need $\mu \geq 1$. We find also that $\frac{d}{d x} F_{\mu}(0)=\mu$ and $\frac{d}{d x} F_{\mu}\left(x^{*}(\mu)\right)=2-\mu$. So 0 is always unstable (since $\mu \geq 1$ ) and $x^{*}(\mu)$ is stable only for $1<\mu<3$. There is a saddle-node bifurcation at $\mu=1$ when $x=0$ and $x^{*}(\mu)$ annihilate each other. At $\mu_{0}=3$, $\frac{d}{d x} F_{\mu}\left(x^{*}\left(\mu_{0}\right)\right)=-1$, so $x^{*}(\mu)$ undergoes a pitchfork (period doubling) bifurcation and becomes unstable for $\mu \geq 3$. Moreover, a stable 2 -cycle ${ }^{10}$ is born. The two period 2 periodic points $x_{1}, x_{2}$ satisfy the equations

$$
\begin{aligned}
& x_{2}=F_{\mu}\left(x_{1}\right)=\mu x_{1}\left(1-x_{1}\right) \\
& x_{1}=F_{\mu}\left(x_{2}\right)=\mu x_{2}\left(1-x_{2}\right)
\end{aligned}
$$

resulting in

$$
x_{1,2}=\frac{1+\mu \pm \sqrt{\mu^{2}-2 \mu-3}}{2 \mu} .
$$

Since $x_{1}, x_{2}$ form a 2 -cycle for the map $F_{\mu}$, each is a fixed point for the iterated map $F_{\mu}^{2}$. Also the term $\mu^{2}-2 \mu-3$

${ }^{9} \mathrm{~A}$ periodic point of period $n$ for a map $f$ is a point $x_{0}$ which satisfies $f^{n}\left(x_{0}\right)=x_{0}$, where $f^{n}=f \circ \cdots \circ f$ ( $n$ times). If $n$ is a period for $x_{0}$, then $k n$ is also a period for $x_{0}, k=1,2, \cdots$. The smallest of all periods for $x_{0}$ is called the fundamental or prime period.

${ }^{10} \mathrm{~A} m$-cycle for a map $f$ is a set of $m$ distinct points $\left\{x_{1}, \cdots, x_{m}\right\}$ such that $f\left(x_{i}\right)=x_{i+1}, 1 \leq i \leq m-1$, and $f\left(x_{m}\right)=x_{1}$. Hence each $x_{i}$ is a periodic point of period $m$ for $f$. 
in (2) is negative for $\mu<3$, which explains why period 2 fixed points do not exist for $\mu<3$.

Case 3: [Invariant tori] $\left|\lambda_{1}\left(\mu_{0}\right)\right|=\left|\lambda_{2}\left(\mu_{0}\right)\right|=1$, $\operatorname{Im}\left(\lambda_{1}\left(\mu_{0}\right)\right)=-\operatorname{Im}\left(\lambda_{2}\left(\mu_{0}\right)\right)>0$ The implicit function theorem gives a smooth curve $\mu \rightarrow p(\mu)$ through $\left(p_{0}, \mu_{0}\right)$. However, the fixed point solution $p(\mu)$ undergoes a bifurcation at $\mu_{0}$ with the emergence of a continuous one-parameter family of circles $C_{\mu}$ around $p(\mu)$, for $\mu \in\left(\mu_{0}, \mu_{0}+\epsilon\right)$ and small $\epsilon>0$. The circle $C_{\mu}$ is invariant ${ }^{11}$ under the Poincaré map $\mathcal{P}_{\mu}$, hence the forward trajectories $\bigcup_{t>0} \phi_{t}^{\mu}\left(C_{\mu}\right)$ form an invariant 2-dimensional torus for the flow of $\dot{x}=f_{\mu}(x)$. So if $\Gamma$ is the periodic orbit being considered before the bifurcation, then after the bifurcation there are orbits emerging around $\Gamma$ which form a thin torus. We call this a secondary Hopf bifurcation ${ }^{12}$ for the periodic orbit $\Gamma$. Similar to the Hopf bifurcation for an equilibrium solution in the continuous case, a secondary Hopf bifurcation is supercritical (subcritical) if the invariant circle is attracting (repelling) under the flow $\phi_{t}^{\mu}$, and $\Gamma$ becomes unstable (stable).

Invariant tori have recently been detected for a 39-bus power system in [11].

\section{B. Period Doubling as a Route to Chaos}

The classic example of a cascade of period doubling bifurcations leading to chaos is the logistic map $F_{\mu}$ defined in (1). Recall that $F_{\mu}$ has a fixed point given by $x^{*}(\mu)=$ $\frac{\mu-1}{\mu}$. Initially the attracting set consists of the single point $x^{*}(\mu)$ that bifurcates into two points $x_{1,2}(\mu)$ at $\mu_{1}=3.0$, where

$$
x_{1,2}(\mu)=\frac{1+\mu \pm \sqrt{\mu^{2}-2 \mu-3}}{2 \mu} \quad, \quad \mu \geq \mu_{1} .
$$

A simple calculation shows that $\frac{d}{d x} F_{\mu}^{2}\left(x_{1}(\mu)\right)=$ $\frac{d}{d x} F_{\mu}^{2}\left(x_{2}(\mu)\right)=-1$ when $\mu=\mu_{2}=1+\sqrt{6}=3.44949 \cdots$. So the map $F_{\mu}^{2}$ undergoes a period doubling bifurcation at $\mu_{2}$. Since both $x_{1}(\mu), x_{2}(\mu)$ are fixed points for $F_{\mu}^{2}$, they lose their stability and bifurcate into four points at $\mu_{2}$. These four points are stable fixed points for the iterated map $F_{\mu}^{4}$, and thus are stable periodic points of period 4 for $F_{\mu}$. For example, at $\mu=3.5$, the steady state solution (or attracting set) alternates between the four values of $0.82694,0.50088,0.87500$, and 0.38282 . As we increase $\mu$ further, the number of alternating steady state values increases with $2^{n}$, the interval between successive bifurcation values decreases, and the distance between neighboring periodic points decreases until eventually what looks like a chaotic attracting set appears. This is called a period doubling cascade to chaos. The chaotic region is interspersed with bands. In the bands only a small number of periodic points form the attracting set. We

\footnotetext{
${ }^{11}$ A set $Y \subset X$ is invariant under a map $f: X \rightarrow X$ if $f(y) \in Y$ for all $y \in Y$.

${ }^{12}$ In proving the existence of invariant circles $C_{\mu}$, we need to assume the eigenvalue $\lambda_{1}\left(\mu_{0}\right)$ is not a $k^{t h}$ root of unity, $k=1,2, \cdots, 5$. That is, $e^{j k \lambda_{1}\left(\mu_{0}\right)} \neq 1, k=1,2, \cdots, 5$. A proof of the result can be found in [25].
}

Table 1 First Eight Bifurcation Values for the Logistic Map $F_{\mu}$

\begin{tabular}{c|l}
$\mu_{1}=3.0$ & $\mu_{5}=3.568759 \ldots$ \\
$\mu_{2}=3.449490 \ldots$ & $\mu_{6}=3.569692 \ldots$ \\
$\mu_{3}=3.544090 \ldots$ & $\mu_{7}=3.569891 \ldots$ \\
$\mu_{4}=3.564407 \ldots$ & $\mu_{7}=3.569934 \ldots$
\end{tabular}

will discuss how chaos disappears in Section III. Table 1 lists the first eight period doubling bifurcation values for $F_{\mu}$. The sequence of bifurcation values $\left\{\mu_{n}\right\}$ converges geometrically to $\mu_{\infty}=3.569945 \cdots$.

\section{ChaOS, Crises, AND Homoclinic TANGles}

In this section we define chaos and discuss aspects of sudden changes in chaotic attractors which occur as a system parameter is varied. These changes are classified as a boundary crisis or an interior crisis. In the power system example of Section IV, we only find boundary crises. We also discuss homoclinic tangles proposed as models for chaos in power systems in [22] and [30]. Denote a chaotic invariant set by $\Lambda$. The term chaos is associated with the following properties.

1) Sensitivity to initial conditions: Trajectories starting at arbitrarily close initial points will eventually diverge, making prediction impossible.

2) Periodic orbits: $\Lambda$ contains periodic orbits of all periods. It also contains infinitely many nonperiodic orbits.

3) Dense orbit: There is an orbit which is dense in $\Lambda$.

4) Cantor set: $\Lambda$ is a Cantor set.

The last property also implies that $\Lambda$ is a fractal, i.e., a subset of the state space of fractional dimension. ${ }^{13}$ To better understand the dynamics after the onset of chaos, we appeal to a result due to the Russian mathematician Sarkovskii. The result begins with an ordering of the natural numbers. The Sarkovskii ordering of the natural numbers is the following:

$$
\begin{aligned}
& 3 \triangleright 5 \triangleright 7 \triangleright \cdots \\
& 2 * 3 \triangleright 2 * 5 \triangleright 2 * 7 \triangleright \cdots \\
& 2^{2} * 3 \triangleright 2^{2} * 5 \triangleright 2^{2} * 7 \triangleright \cdots \\
& \ldots \ldots \ldots \ldots \ldots \ldots \cdots \cdots \cdots \\
& \ldots \triangleright 2^{5} \triangleright 2^{4} \triangleright 2^{3} \triangleright 2^{2} \triangleright 2 \triangleright 1 .
\end{aligned}
$$

That is, first list all the odd numbers except 1 , followed by 2 times the odds, $2^{2}$ times the odds, $2^{3}$ times the odds, etc. This exhausts all the natural numbers with the exception of the powers of 2 which we list last, in decreasing order.

Theorem 3.1: (Sarkovskii Theorem) Suppose $f: \Re \rightarrow \Re$ is continuous and has a periodic point of prime period ${ }^{14} k$. If $k \triangleright l$ in the Sarkovskii ordering, then $f$ has a periodic point of prime period $l$.

\footnotetext{
${ }^{13}$ See footnote one for the definition of fractional dimension.

${ }^{14} \mathrm{~A}$ point $x_{0}$ is a periodic point of prime period $k$ for a map $f$ if it is a periodic point of period $k$ for $f$ and $k$ is the smallest positive integer for which $f^{k}\left(x_{0}\right)=x_{0}$ holds.
} 
Returning to our logistic map example, we see that for $\mu_{1}<\mu<\mu_{2}$, there are two period 2 points $x_{1}(\mu), x_{2}(\mu)$ given by (3). So by the Sarkovskii theorem there must be a period 1 point (or a fixed point), which we know is $x^{*}(\mu)=\frac{\mu-1}{\mu}$. The point $x^{*}(\mu)$ is unstable ${ }^{15}$ for $\mu \geq \mu_{1}$. Sarkovskii's theorem implies that as $\mu$ is increased, the logistic map $F_{\mu}$ must have periodic points of all periods of the form $2^{n}$ before it can have infinitely many periodic points with distinct periods. It also hints at the possibility of small windows of stable periodic behavior after the onset of chaos. For $\mu \approx 3.83$, there is a stable 3-cycle. So Sarkovskii's theorem implies there are periodic points of all periods. This suggests that after the onset of chaos for the power system example in Section IV there exist small windows of stable periodic orbits that are not easily detected by numerical experiments.

The bifurcation diagram for the logistic map is interspersed with bands, and in the bands only a small number of periodic points form the attracting set. So there is a transition from a chaotic attracting set containing infinitely many periodic points to an attracting set with only a small number of periodic points. The simulations in Section IV reveal the annihilation of the chaotic attractor as the reactive power at the load bus is increased. Next, we discuss "crises" involving chaotic attractors. These phenomena characterize when and how a chaotic attractor is destroyed. To understand how the chaotic attractor vanishes, we first consider an equivalent form of the logistic map. The logistic map $F_{\mu}(x)=\mu x(1-x)$ can be transformed to a quadratic map $G_{c}(y):=c-y^{2}$ via the coordinate change $y=\mu x-\frac{\mu}{2}$ and the parameter change $c=\frac{\mu^{2}}{4}-\frac{\mu}{2}$ (or equivalently, $\mu=1+2 \sqrt{\left.\frac{1}{4}+c\right)}$. There are good reasons to call $G_{c}(y)$ the logistic map. For example, the Henon map $I I(x, y)$ defined by

$$
H:\left[\begin{array}{l}
x \\
y
\end{array}\right] \mapsto\left[\begin{array}{c}
y \\
c-y^{2}+b x
\end{array}\right], b \geq 0
$$

reduces to $G_{c}(y)$ when $b \downarrow 0$. Also, for small values of $b$, the dynamics of $H(x, y)$ becomes invariant on the manifold $\left\{(x, y): y=c-x^{2}\right\}$, which may explain why the shape of the chaotic attractor of the Henon map resembles a quadratic curve. ${ }^{16}$ More importantly, the simple form of $G_{c}(y)$ makes the computation of bifurcations and basins of attraction of fixed points much easier.

Since $1 \leq \mu \leq 4$ for $F_{\mu}$, the parameter change implies $-\frac{1}{4} \leq c \leq 2$. For $c<-\frac{1}{4}$. no fixed point exists and all trajectories go to $-\infty$. At $c=-\frac{1}{4}$ a saddlenode bifurcation occurs. The bifurcation creates a pair of stable and unstable fixed points. These fixed points are $y_{u}(c)=-\frac{1}{2}-\sqrt{\frac{1}{4}+c}$ which is unstable, and $y_{s}(c)=$

\footnotetext{
${ }^{15}$ The Sarkovskii theorem does not predict which periodic points are stable.

${ }^{16}$ We will discuss in Section IV-D that the chaotic attractor of the power system model is "close" to the constraint manifold obtained by treating the load variables as very fast system variables. An explanation for that phenomenon may help us to understand why the chaotic attractor of the Henon map is "close" to and resembles the quadratic curve.
}

$-\frac{1}{2}+\sqrt{\frac{1}{4}+c}$ which is stable. ${ }^{17}$ As $c$ is increased to 0.75 (corresponding to $\mu=3.0$ ), $y_{s}$ (c) undergoes a period. doubling bifurcation, followed by a cascade of period doubling leading to chaos.

In Section II we reviewed bifurcations of equilibrium points and periodic orbits. Here we draw comparisons between bifurcation of a fixed point and that of a chaotic attractor. A simple definition of a crisis [17] is as follows: A crisis is a collision between a chaotic attractor and a coexisting unstable fixed point or periodic orbit. Much of the classification of a crisis given below is the result of extensive numerical experiments on the quadratic map $G_{c}(y)$ in [17].

- Boundary crisis: The sudden destruction of the chaotic attractor and its basin of attraction is symptomatic of a boundary crisis. For parameter values just past the crisis point, the attractor no longer exists. Trajectories starting in the region that was occupied by the destroyed attractor would appear to be chaotic, in a fashion similar to what it was before the crisis, but only for a finite time after which the trajectory would leave the region. One is led to speculate what happens after the destruction of the chaotic attractor in the power system model to be considered in Section IV. One possible explanation is that an unstable voltage trajectory may "wander" in the vacancy created by the destroyed attractor for a long time before escaping to $\pm \infty$. For the quadratic map $G_{c}(y)$, the unstable fixed point $y_{u}$ is on the boundary of the basin of attraction when $c=2$. As $c$ is increased beyond 2, the chaotic attractor is destroyed and all trajectories diverge to $-\infty$. A boundary crisis occurs when the unstable limit set, such as a saddle periodic orbit, that collides with the chaotic attractor is on the boundary of the basin of attraction of the attractor.

- Interior crisis: This is different from the boundary crisis because collision occurs within the basin of attraction. Consider the map $G_{c}(y)$. A bifurcation at $c=1.755$ leads to the birth of a stable 3 -cycle and an unstable 3-cycle. The stable 3-cycle becomes chaotic via period doubling. Since each individual "branch" of the 3-cycle undergoes period doubling, the chaotic attractor lies in three pieces. At $c \approx 1.79$ the unstable 3-cycle collides with the three-piece attractor. The three chaotic bands widen to form a single band. An interior crisis is signified by a discontinuous widening of the attractor.

Fix $b$ at 0.3 and vary $c$. As $c$ is increased; period doubling of the stable 6 -cycle leads to chaos. At $c \approx 1.0807$ a boundary crisis and therefore a homoclinic tangency ${ }^{18}$ occurs

\footnotetext{
${ }^{17}$ They correspond to the unstable fixed point $x=0$ and stable fixed point $x^{*}(\mu)=\frac{\mu-1}{\mu}$ for the map $F_{\mu}$.

${ }^{18}$ Let $p$ be a saddle equilibrium point (fixed point) for a differential equation $\dot{x}=f(x)$ (a map $x \mapsto f(x)$ ). Suppose its stable manifold $W^{s}(p)$ intersects transversely with its unstable manifold $W^{u}(p)$ at a point $q$, i.e., the tangents to the two manifolds at $q$ span the state space The point $q$ is a homoclinic point, or $q$ is said to be homoclinic to $p$. The coincidence of $W^{u}(p)$ and $W^{3}(p)$ is known as a homoclinic tangency.
} 
when the unstable 6-cycle on the boundary collides with the chaotic attractor. One can verify that the unstable 6-cycle is on the boundary by observing whether some component of its unstable manifold converges to the attractor. Unlike $G_{c}(y), H(x, y)$ is a diffeomorphism. ${ }^{19}$

Consider a chaotic attractor which emerges as a result of a period doubling cascade. Are period doubling cascades prerequisites for homoclinic tangles $?^{20}$ From the definition of a boundary crisis, it is clear that a homoclinic tangency is a precursor to a boundary crisis. It seems plausible to assign the following sequence to these global bifurcations: ${ }^{21}$

$$
\begin{gathered}
\text { Period doubling } \rightarrow \text { Chaos } \rightarrow \text { Homoclinic tangles } \\
\rightarrow \text { Homoclinic tangency } \rightarrow \text { Boundary crisis. }
\end{gathered}
$$

Suppose one has an explicit expression for a homoclinic orbit. Then the method of Melnikov, which uses perturbation analysis about the homoclinic orbit, can be used to prove the existence of homoclinic tangles. A classical three node power system where the mass of one generator is considerably larger than the other generator, thereby reducing the system to Sitnikov's three body problem, is analyzed in [22]. A classical power system, without generator damping, has a Hamiltonian structure. For such a system, it is shown in [30] that a form of chaos called Arnold diffusion can occur. Both studies use Melnikov's method. It is not possible to use Melnikov's method for the model power system in our work, because for the example in Section IV we do not have an explicit expression for the homoclinic orbit.

\section{Simulation Results for a Power System Model}

A bifurcation analysis of a three-bus power system model, shown in Fig. 1 and previously proposed in [14] and [3], is presented. The bifurcation diagrams show that the system exhibits both static and dynamic bifurcations, and cascades of period doubling bifurcations leading to chaos. We also study the effects of these bifurcations on system trajectories by investigating the behavior of the trajectories near the stable operating point for parameter values corresponding to the various types of bifurcation.

\section{A. System Model}

The system model consists of a load bus and two generator buses, one of which is a slack bus (infinite bus). The other generator bus has a fixed voltage magnitude and swing equation dynamics as given below in (5) and (6). The load is modeled as an induction motor.

\footnotetext{
${ }^{19} \mathrm{~A}$ diffeomorphism is a differentiable map whose inverse exists and is also differentiable. The Henon map $H(x, y)$ is an area contracting diffeomorphism if $|b|<1$

${ }^{20} \mathrm{~A}$ homoclinic tangle is a set of homoclinic points.

${ }^{21}$ The order of occurrence depends on the direction in which the parameter is varied.
}

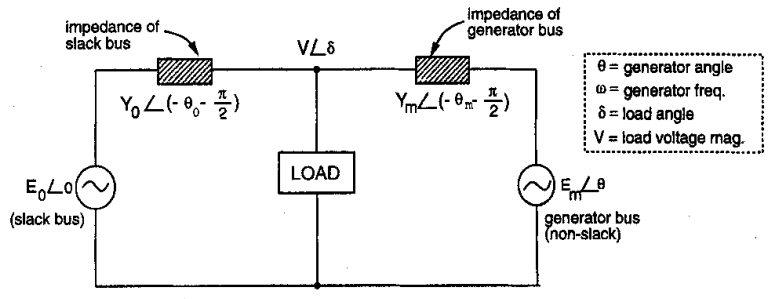

Fig. 1. A three-node power system model.

We use the parameter values in [3]. This gives rise to a system of four differential equations of the form:

$$
\begin{aligned}
\dot{\theta} & =\omega \\
\dot{\omega} & =-d_{m} \omega-V \sin (\theta-\delta)+P_{m} \\
K_{q w} \dot{\delta} & =-K_{v_{2}} V^{2}-K_{q v} V+Q_{e}-Q \\
& =: g_{1}(\theta, \delta, V ; Q) \\
K_{q w} K_{p v} T \dot{V} & =K_{p w}\left[K_{v_{2}} V^{2}+K_{q v} V-Q_{e}+Q\right] \\
& +K_{q w}\left[P_{e}-P-K_{p v} V\right] \\
& =: g_{2}(\theta, \delta, V ; Q) .
\end{aligned}
$$

The variables $\theta$ and $\omega$ are the machine angle (in radians) and angular velocity (in radians per second). $V$ and $\delta$ are the voltage magnitude (in pu) and angle of the load bus. The normalized nonslack generator damping coefficient is $d_{m}$. The terms in square brackets are the load flow equations for a constant PQ load, where $P$ and $Q$ are the real and reactive power demand at the load bus. The electrical real power $P_{e}$ and reactive power $Q_{e}$ delivered to the load are

$$
\begin{aligned}
& P_{e}=-\gamma_{1} V \sin (\delta)-\gamma_{2} V \sin (\delta-\theta) \\
& Q_{e}=+\gamma_{1} V \cos (\delta)+\gamma_{2} V \cos (\delta-\theta)-\gamma_{3} V^{2}
\end{aligned}
$$

where $\gamma_{i}$ are products of conductances and constant voltages behind reactance. The coefficients $K_{i j}$ are derived from the induction motor model proposed in [14] and [3]. The constant $K_{q w}$ is small so that the $(\delta, V)$ dynamics are faster than that of $(\theta, \omega)$. The real and reactive powers $P_{e}$ and $Q_{e}$ demanded by the induction motor are modeled as

$$
\begin{aligned}
P_{e} & =K_{p w} \dot{\delta}+K_{p v} V+K_{p v} T \dot{V}+P \\
Q_{e} & =K_{q w} \dot{\delta}+K_{q v} V+K_{v_{2}} V^{2}+Q .
\end{aligned}
$$

Note that (12) is the same as (7). The variable $\dot{\delta}$ can be eliminated by substituting (12) into (11) to obtain (8).

\section{B. Bifurcation Analysis}

Three sets of bifurcation diagrams are drawn for different values of machine damping coefficient $d_{m}$. The parameter varied in each diagram is $Q$, the reactive power demand. The bifurcation diagram that exhibits the richest qualitative behavior, including two period-doubling routes to chaos, is for the value $d_{m}=0.05$. When $d_{m}$ is increased to 0.1 , 


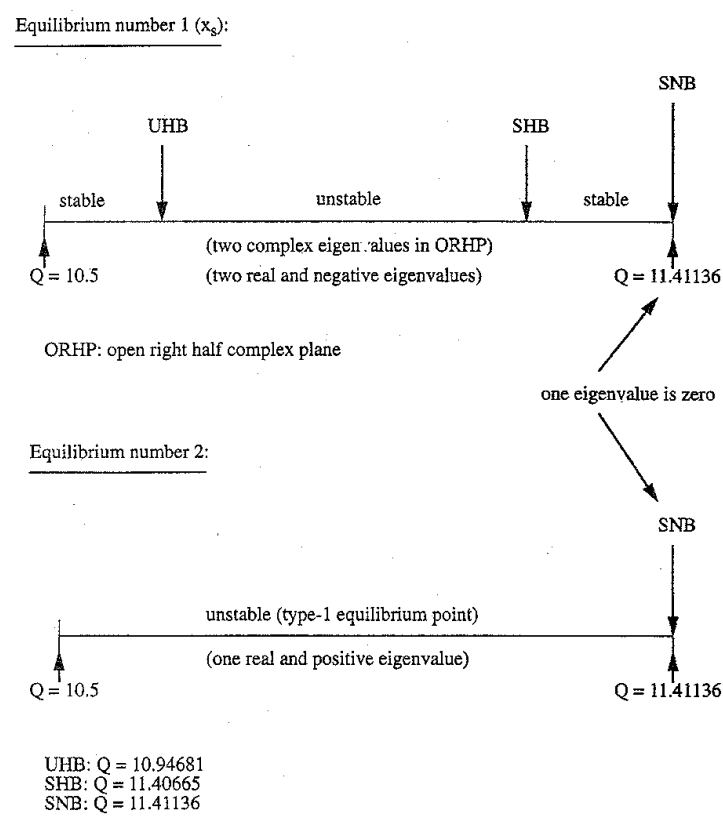

Fig. 2. Stability of equilibrium points showing local bifurcation values.

all period-doubling bifurcations are suppressed. At $d_{m}=$ 0.114 the only bifurcation is the saddle-node bifurcation (SNB), defined in Section I. For $d_{m}=0.05$ we observe six types of bifurcations:

1) CFB, Cyclic Fold Bifurcation: $Q=10.81813$,

2) $P D B_{1}$, Period Doubling Bifurcation: $Q=10.87327$,

3) UHB, Unstable or Subcritical Hopf Bifurcation: $Q=$ 10.94681 ,

4) $P D B_{2}$, Period Doubling Bifurcation: $Q=11.38779$,

5) $S H B$, Stable or Supercritical Hopf Bifurcation: $Q=$ 11.40665 ,

6) $S N B$, Saddle-Node Bifurcation: $Q=11.41136$.

All the simulation results are obtained using the bifurcation analysis software package AUTO [9] and the nonlinear system simulation toolkit DSTOOL [7]. ${ }^{22}$

Fig. 2 shows the location of the Hopf bifurcations with respect to the position of the eigenvalues for $d_{m}=0.05$. For the range of $Q$ values considered, the system has two equilibrium points. One of them is always unstable. The Jacobian at this equilibrium has one real and positive eigenvalue and three open left half plane eigenvalues, so it is a type-1 unstable equilibrium. The other equilibrium. is stable and denoted $x_{s}$. At $Q=10.94681$, there is a $U H B$ with the emergence of an unstable limit cycle around $x_{s}$ for $Q<Q_{U H B}$, hence by the exchange of stability property given in [25], $x_{s}$ loses stability for $Q>Q_{U H B}$. At $Q=11.40665$, there is a $S H B$, so $x_{s}$ regains stability for $Q>Q_{S H B}$. At $Q=11.41136$, a $S N B$ occurs and the equilibria coalesce and become a single equilibrium at which the Jacobian is singular and has a simple zero eigenvalue.

\footnotetext{
${ }^{22}$ For details, email the authors at dstool-list@macomb.tn.comell.edu.
}
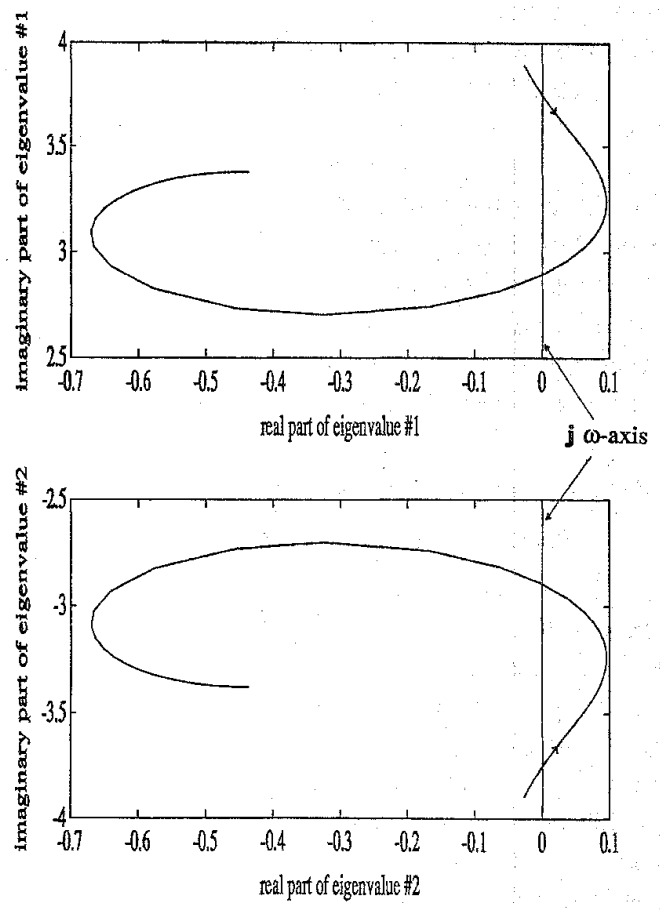

Fig. 3. Location of the complex eigenvalues as $Q$ changes.

The Jacobian at $x_{s}$ has a pair of complex eigenvalues and two real and negative eigenvalues. Fig. 3 shows the movement of the pair of complex eigenvalues, as $Q$ is increased from 10.5 to $Q_{S N B}=11.41136$. The eigenvalues cross the $j \omega$ axis transversely at $Q=Q_{U H B}$ so that $x_{s}$ becomes unstable for $Q>Q_{U H B}$, and recross it at $Q=Q_{S H B}$ so that $x_{s}$ regains stability for $Q>Q_{S H B}$. At $Q=Q_{U H B}$, the complex eigenvalues are $\pm j \omega_{0}=$ $\pm j 3.746$. By the Hopf bifurcation theorem, the unstable. oscillations corresponding to $Q$ values slightly less than $Q_{U H B}$ have periods approximately equal to $\frac{2 \pi}{\omega_{0}}$. We check that for $Q=10.9459$, a parameter value slightly less than $Q_{U H B}=10.94681$, there is an unstable periodic solution oscillating at a period of $T=1.677 \approx \frac{2 \pi}{\omega_{0}}$ seconds. For $Q$ near $Q_{S N B}$, the real part of the eigenvalue is very sensitive to changes in $Q$. At $Q=Q_{S N B}$, one of the two real and negative eigenvalues crosses the $j \omega$ axis and becomes positive. This reactive power demand corresponds to the system steady-state operating limit, and the system has no operating solution for $Q>Q_{S N B}$.

\section{The Effects of Damping on the Occurrence of Bifurcations}

Figs. 4-6 show bifurcation diagrams for three values of machine damping: $\left.\left.d_{m}=1\right) 0.050,2\right) 0.100$, or 3) 0.114 . From (5)-(8) we know that different values of $d_{m}$ have no effects on the locations of the two equilibrium points. However, the Jacobian evaluated at the equilibrium points will be different. In Fig. 5 there are no period doubling bifurcations. Also implied is the absence of chaos, since the route to chaos (for the system being considered here) is followed via a sequence of period doubling bifurcations as 


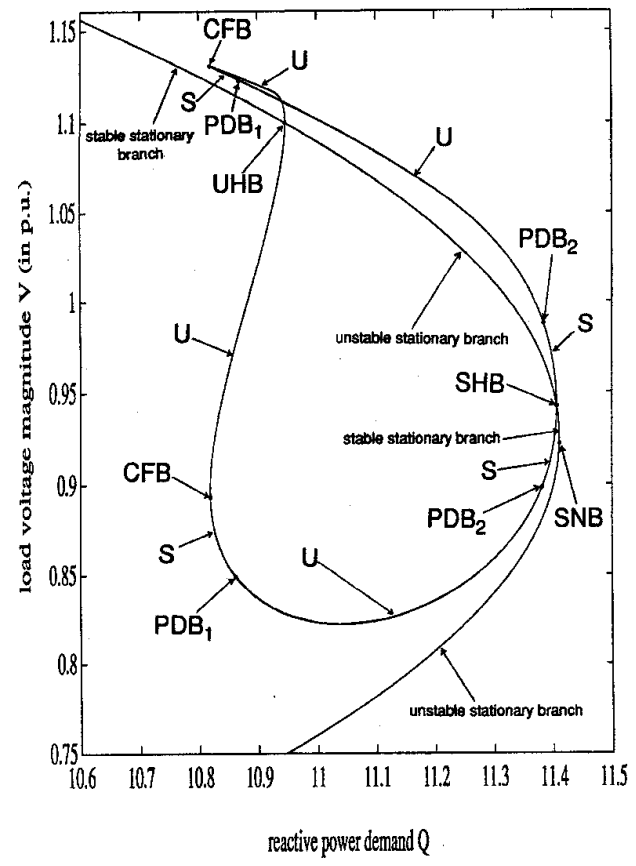

S: stable periodic branch

U: unstable periodic branch

Fig. 4. Bifurcation values for $\mathrm{V}$, damping $=0.05$.

will be seen later. It may be possible as in [4] to calculate the value of $d_{m}$ at which no period doubling bifurcation can occur. Fig. 5 also shows that a UHB occurs at $Q=11.3365$ and a SHB occurs at $Q=11.3975$. The unstable and stable limit cycles emerging from these two bifurcations collide at a CFB at $Q=11.3295$. Also, the distance between the two values $Q_{U H B}$ and $Q_{S H B}$ for $d_{m}=0.1$ is smaller than that for $d_{m}=0.05$. In Fig. 6 , all the dynamic bifurcations $C F B, U H B$ and $S H B$ disappear. There is only a static bifurcation SNB at $Q=11.41136$. In fact, as $d_{m}$ is increased, $U H B$ and $S H B$ will come closer until they coincide with each other and disappear at some value $Q=Q_{H B}$. This corresponds to a degenerate Hopf bifurcation [10] and an eigenvalue plot similar to that in Fig. 3 will show a nontransversality in the pair of eigenvalues crossing the $j \omega$ axis.

\section{Period Doubling Routes to Chaos}

For $d_{m}=0.05$, numerical results show that there is cascade of period doubling bifurcations starting at $Q_{P D B_{1}}=$ 10.87327. Similar to that for the logistic map discussed in Section II, this sequence of bifurcation values converges quickly to a value around 10.89. Fig. 7 shows the bifurcation into doubly periodic oscillations in state space and Fig. 8 shows voltage magnitude versus time of the period doubled bifurcation. A periodic double-loop is clearly observed in Fig. 7. Fig. 8 is the corresponding plot of $V$ versus time and shows that the "lower envelope" exhibits modulation between the two values 0.83 and 0.855 . The period of this periodic double-loop is $3.31 \mathrm{~s}$, roughly

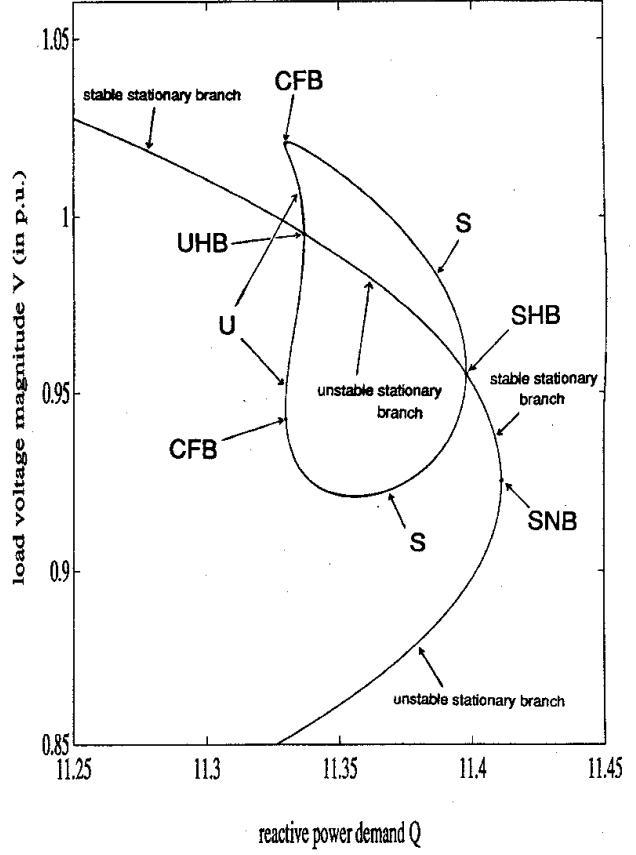

S: stable periodic branch

U: unstable periodic branch

Fig. 5. Bifurcation values for $\mathrm{V}$, damping $=0.1$.

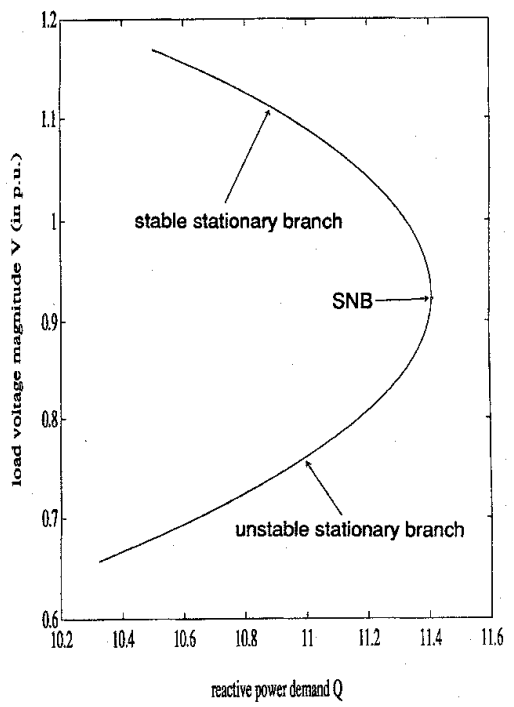

Fig. 6. Bifurcation values for $\mathrm{V}$, damping $=0.114$.

twice the "fundamental period" $\frac{2 \pi}{\omega_{0}}$, where $\omega_{0}=3.78$ is the imaginary part of the pair of complex eigenvalues at $Q=10.87857$. Further period doubling bifurcations occur as $Q$ is increased. These period doublings accumulate in a dense fashion on the attractor shown in Fig. 9 for $Q=10.894$. The chaotic motion has a crude orbit structure, and its "fundamental period" is approximately $1.67 \mathrm{~s}$. As implied by the Silnikov theorem [18], this period is roughly $\frac{2 \pi}{\omega_{1}}$, where $\omega_{1}=3.771$ is the imaginary part of the complex eigenvalues at $Q=10.894$. Therefore the approximate 


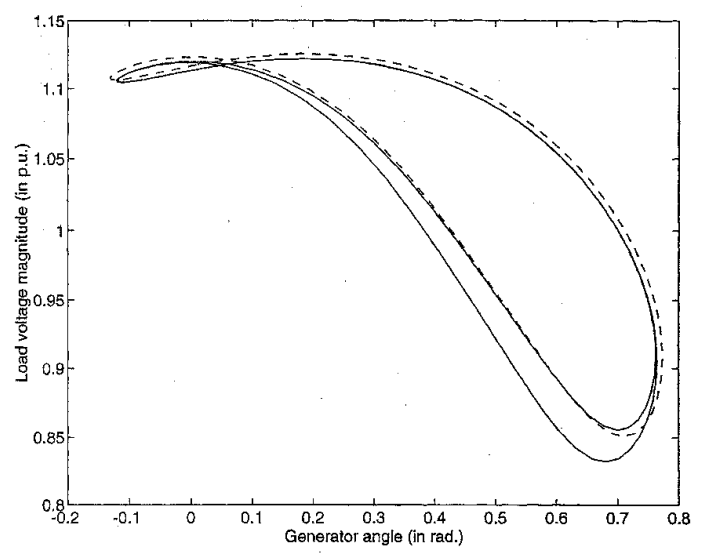

Fig. 7. Bifurcation of a periodic solution (dashed) into a doubly periodic oscillation (solid), $Q=10.87857$.

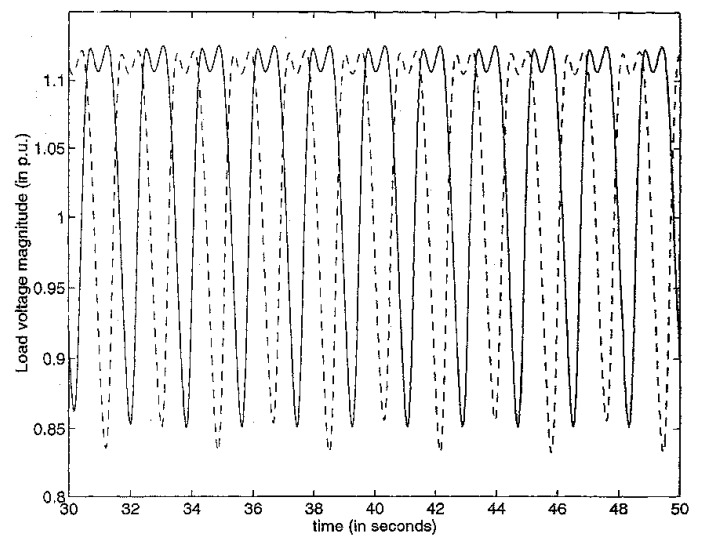

Fig. 8. Voltage versus time of a periodic solution (solid) and its doubly periodic oscillation after a PDB (dashed), $Q=10.87857$.

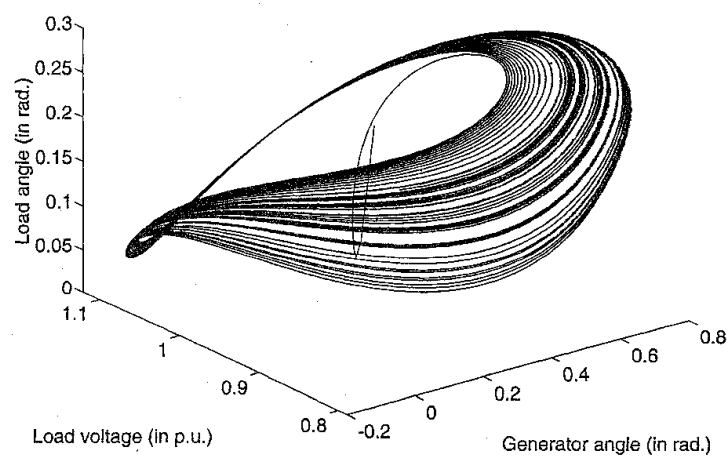

Fig. 9. Chaotic dynamics due to successive PDB's, $Q=10.894$.

frequency of the chaotic oscillation is between 1 and $2 \mathrm{~Hz}$. The chaotic structure is first observe at about $Q=10.89$, but disappears in a boundary crisis when the reactive power demand is increased to $Q=10.894$.

When $Q$ is decreased beyond $P D B_{2}$, there is a similar cascade of period doubling bifurcations. Similar to the other chaotic attractor that exists for $10.89 \leq Q \leq 10.894$, this attractor exists for $Q$ in the range [11.377, 11.38]. We observed that the strange attractor at $Q=10.894$ is larger

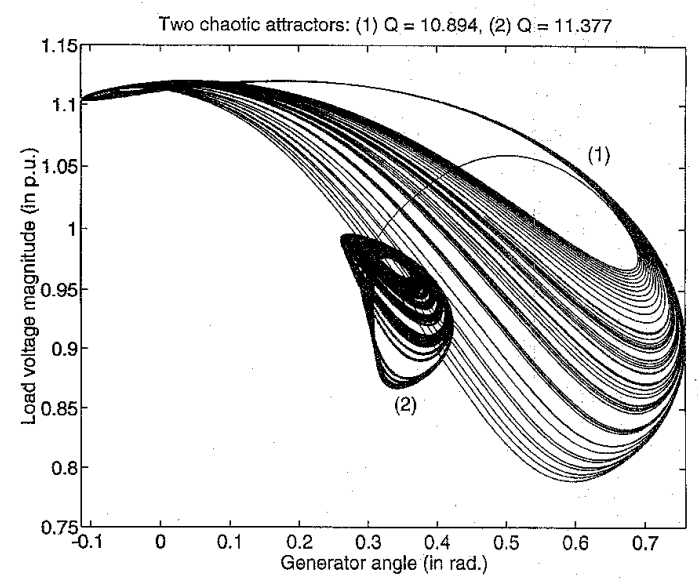

Fig. 10. The two chaotic attractors: (1) $Q=10.894$, and (2) $Q=11.377$.

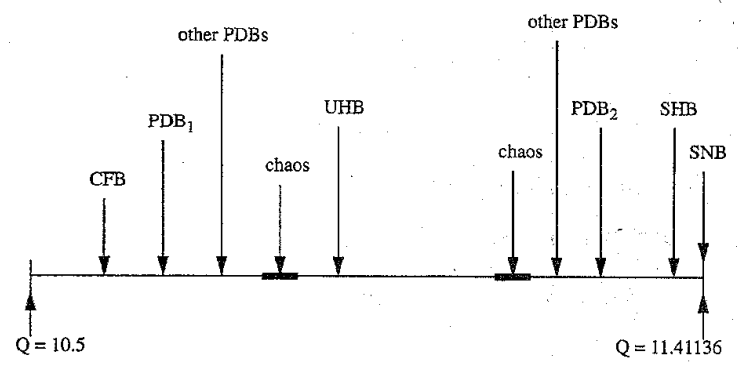

CFB: $Q=10.81813, P^{2} D_{1}: Q=10.87327, \quad$ UHB: $Q=10.94681$

chaos: $10.89 \leq \mathrm{Q} \leq 10.894$

SNB: $Q=11.41136$, SHB: $Q=11.40665, \quad P B D_{2}: Q=11.38779$

chaos: $11.377 \leq \mathrm{Q} \leq 11.38$

Fig. 11. Partition of the parameter space with respect to bifurcation values.

in size than the one obtained for $Q=11.377$. Fig. 10 shows a comparison of the two attractors in the $(\theta, V)$ space. Finally, Fig. 11 shows the partition of the parameter space, $10.5 \leq Q \leq 11.41136=Q_{S N B}$, with respect to the various bifurcation values considered in this section.

\section{E. Chaotic Dynamics and the Constraint Manifold}

Suppose we set $K_{q w}=0$ in (7) and (8) so that the load variables $(\delta, V)$ are infinitely fast. Then the system equations will consist of two differential equations constrained by two algebraic equations:

$$
\begin{aligned}
\dot{\theta} & =\omega \\
\dot{\omega} & =-d_{m} \omega-V \sin (\theta-\delta)+P_{m} \\
0 & =g_{1}(\theta, \delta, V ; Q) \\
0 & =g_{2}(\theta, \delta, V ; Q)
\end{aligned}
$$

where $g_{1}(\theta, \delta, V ; Q)$ and $g_{2}(\theta, \delta, V ; Q)$ are the right-hand sides of (7) and (8), respectively. The dynamics of (13)-(16) 


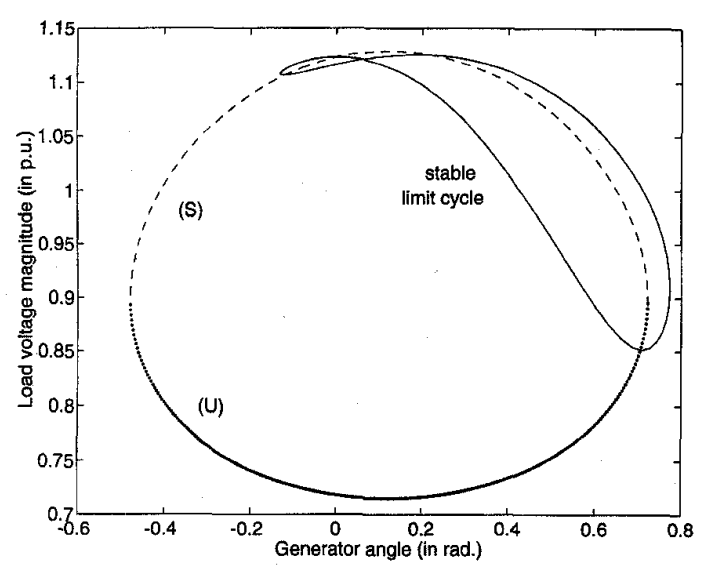

Fig. 12. Stable limit cycle and constraint manifold, $Q=10.85582$. In this and later figures, (S) and (U) denote the stable and unstable components of the constraint manifold.

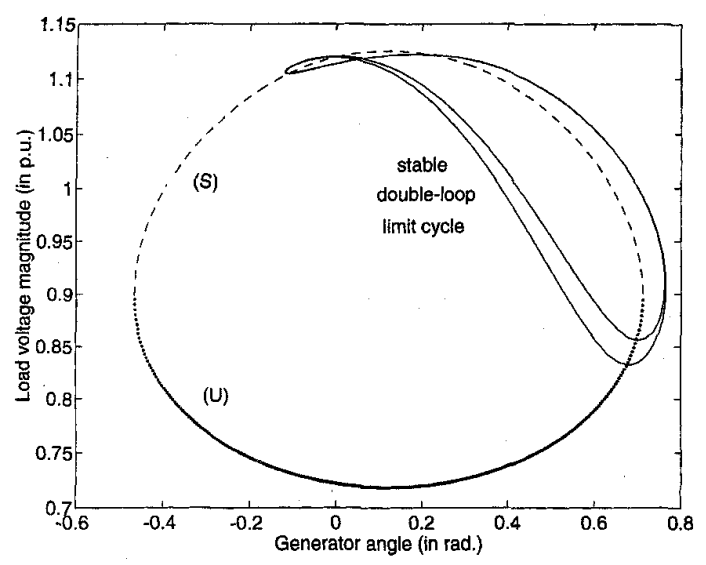

Fig. 13. Stable double-loop limit cycle and constraint manifold, $Q=10.87858$.

are confined to lie on the two-dimensional constraint manifold

$$
M:=\left\{(\theta, \omega, \delta, V): g_{1}(\theta, \delta, V ; Q)=g_{2}(\theta, \delta, V ; Q)=0\right\} .
$$

A companion paper in this session discusses systems with constraints. The model given by (5)-(8), however, admits a realistic, small positive parameter $K_{q w}$. For nonzero $K_{q w}$, it is plausible that singular perturbation results in [13] and [29] can be modified to show that trajectories of (5)-(8) are close to the stable component ${ }^{23}$ (denoted $M_{s}$ ) of $M$. We postulate that trajectories going through period doubling bifurcations will lie close to the stable component of $M$, and will be repelled from the unstable component (denoted $M_{u}$ ).

When $Q=10.85582$, a value smaller than $Q_{P D B_{1}}$, Fig. 12 shows $M$ and the stable limit cycle $\Gamma_{s}$ and it can be seen that $\Gamma_{s}$ lies close to $M_{s}$. As $Q$ is increased beyond $Q_{P D B_{1}}$, Fig. 13 shows that a stable double-loop limit cycle

\footnotetext{
${ }^{23}$ The stable (unstable) component of $M$ is the set of points $(\theta, \omega, \delta, V) \in M$ where all (some) eigenvalues of $\left[D_{(\delta, V)} g_{1}(\theta, \delta, V ; Q) \quad \mid \quad D_{(\delta, V)} g_{2}(\theta, \delta, V ; Q)\right]$ are in the open left-half (right-half) complex plane.
}

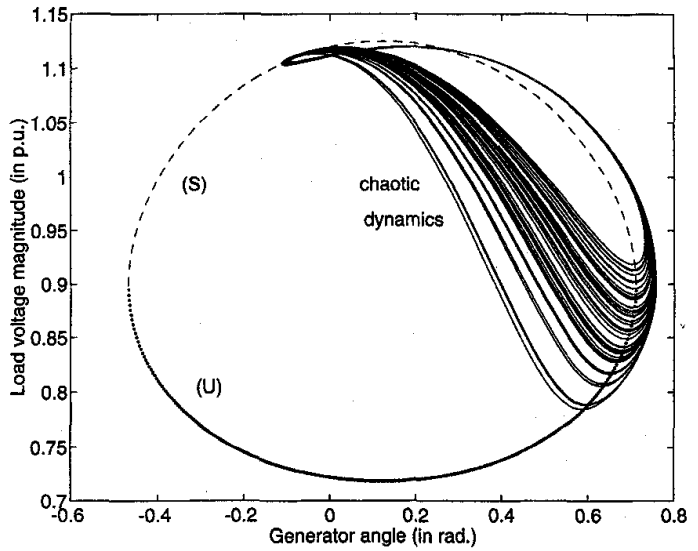

Fig. 14. Chaotic trajectory and constraint manifold, $Q=10.894$.

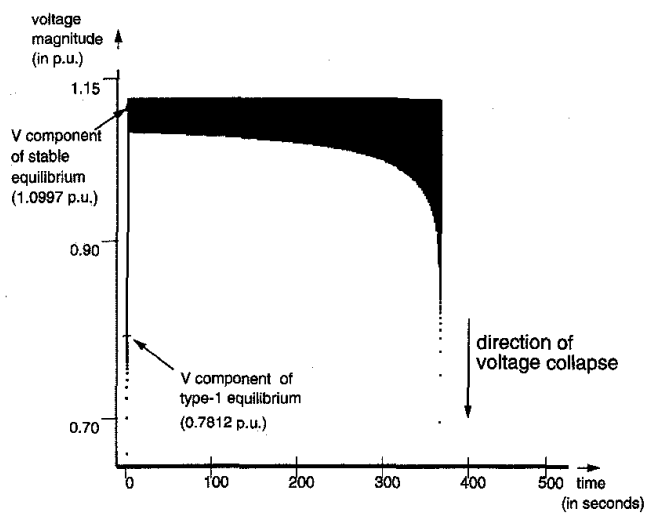

Fig. 15. Voltage collapse: divergence of the unstable manifold of the type- 1 equilibrium, $Q=10.945$.

is born. The two loops "collapse" into a single loop at points near $M_{s}$. The same is true as $Q$ is further increased. Fig. 14 shows that period doubling bifurcations result in a "thickening" of the trajectory at points close to $M_{u}$. The orbits appear to collapse into one loop near $M_{s}$. These observations are yet to be mathematicized.

\section{F. Voltage Collapse}

For some values of $Q$ smaller than $Q_{U H B}=10.94681$ but larger than $Q=10.894$ at which chaos gets annihilated, the unstable manifold of the type- 1 equilibrium does not converge to $x_{s}$. Fig. 15 shows that for $Q=10.945$, the voltage component of the unstable manifold oscillates around and away from the stable equilibrium $x_{s}$, and later experiences a sudden sharp drop-a voltage collapse. Fig. 16, for $Q=11.378$, shows that the voltage component of the unstable manifold of the type- 1 equilibrium converges to the chaotic attractor. The presence of this stable structure (the chaotic attractor) appears to "stabilize" the system, preventing voltage collapse. Increasing $Q$ after encountering the second chaotic attractor, we observe inverse period doubling at $Q=11.38779$. This culminates in a supercritical Hopf bifurcation at $Q=11.40665$. The saddle-node bifurcation occurs at $Q=11.41136$. 


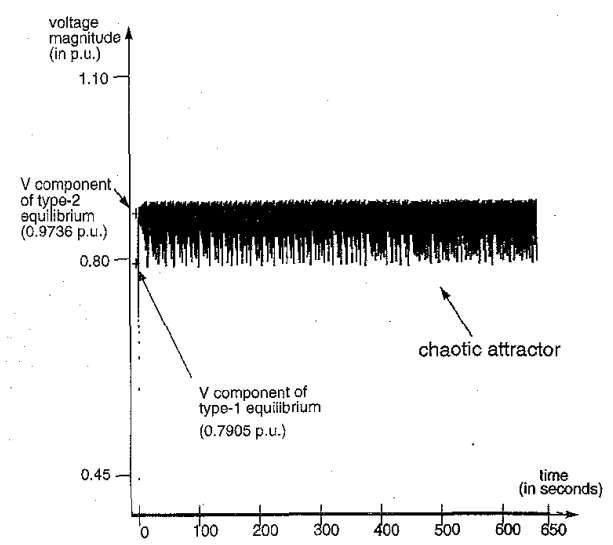

Fig. 16. Reappearance of chaotic attractor, $Q=11.378$.

\section{CONCLUDING REMARKS}

Bifurcations occur at parameter values where there is a qualitative change in system behavior. For the three-bus model studied in Section IV, this change can characterized in terms of attractors.

1) There are at most three attractors for any value of $Q \in\left[10.5, Q_{S N B}=11.41136\right]$, denoted $R_{1}, R_{2}$ and $R_{3}$. We consider infinity $( \pm \infty)$ to be an attractor. Label $\pm \infty$ as $R_{1}$.

2) The attractor labeled $R_{2}$ is the one of most engineering interest. For $Q<Q_{U H B}$ and $Q>Q_{S H B}$, $R_{2}$ is $x_{s}$, the stable operating point. For values of $Q$ between $Q_{U H B}$ and $Q_{S H B}, R_{2}$ disappears since $x_{s}$ becomes unstable and the Jacobian has a pair of complex open right half plane eigenvalues.

3) $R_{3}$ makes its appearance at $Q=Q_{C F B}$ as a stable limit cycle. $R_{3}$ undergoes several dynamic bifurcations after $Q_{P D B_{1}}$. The period doubling cascade transforms $R_{3}$ into a chaotic attractor; a possible global bifurcation eliminates $R_{3}$ at a $Q$ value near 10.894. $R_{3}$ reappears just before $Q_{P D B_{2}}$ as another chaotic attractor. Between $Q_{P D B_{2}}$ and $Q_{S H B}, R_{3}$ is a stable limit cycle.

4) For values of $Q$ between 10.894 (which corresponds to the disappearance of the first chaotic attractor) and 11.377 (which corresponds to the appearance of the second chaotic attractor), numerical results show that $R_{1}$ is the only attractor. These parameter values imply transient instability. Also voltage collapse can take place.

5) There must exist at least one unstable limit set on the boundary of each of the attractors $R_{i}, 1 \leq i \leq 3$. Denote the boundary of $R_{i}$ by $\partial\left(R_{i}\right)$. We can test this by checking whether some component of the unstable manifold of the unstable limit set converges to $R_{i}$. We restrict ourselves to "some component" because an unstable limit set could be on a boundary $\partial\left(R_{i}\right)$ which is "shared" by more than one attractor.

6) For the example considered in Section IV, the following unstable limits sets, denoted $U_{i}$, can be identified. $U_{1}$ is a type-1 unstable equilibrium point that persists until $Q_{S N B}$. Between $Q_{C F B}$ and $Q_{U H B}$, the unstable limit cycle emerging from the subcritical Hopf bifurcation at $Q_{U H B}$ is labeled as $U_{2}$. Since the two chaotic attractors are formed as a result of two cascades of periodic doubling bifurcations, there is probably a countable but infinite number of unstable limit cycles for $Q$ values belonging to two narrow nonintersecting intervals which lie in between the values $Q_{P D B_{1}}$ and $Q_{P D B_{2}}$. Between $Q_{U H B}$ and $Q_{S H B}, x_{s}$ turns into a type-2 unstable equilibrium point, which we label as $U_{3}$. After $Q_{S H B}$ only $U_{1}$ remains.

7) A component of the unstable manifold of $U_{1}$ converges to $R_{2}$ for $Q \leq 10.894$. The load demand $Q=10.894$ is where the chaotic attractor that occurs after $P D B_{1}$ vanishes. At $Q=11.37$, another chaotic attractor makes its appearance. For $10.894 \leq Q \leq$ 11.37, the same component of the unstable manifold of $U_{1}$ converges to $R_{1}$ (infinity). For $11.37 \leq Q \leq$ 11.38 , this component converges to $R_{3}$, which is now a chaotic attractor. It is clear that for parameter intervals between these "switchings". of convergence to various $R_{i}$, there must exist scenarios that violate transversality. Examples of nontransversal scenarios are trajectories connecting pairs of unstable limit sets.

In seeking explanations other than the saddle-node bifurcation to voltage collapse, we have presented a numerical example in Section IV where voltage collapse occurred because the chaotic attractor is destroyed in a boundary crisis. Prior to voltage collapse, we have identified, using the results of Silnikov in [18], that the approximate frequency of chaos is related to the imaginary part of the complex eigenvalues of the Jacobian linearized about the operating point. This approximate frequency is between 1 and $2 \mathrm{~Hz}$. Whether or not such an oscillation is acceptable to the power system depends on the mechanical frequency of generators connected to the network. Electrical subsynchronous resonances ${ }^{24}$ have been known to damage the mechanical shaft of generators. Although the example presented in Section IV is small, the same conclusions seem to hold for a 39-bus power system [11]. This confirms that Hopf bifurcations and chaos do occur in large scale electric networks operating under stressed conditions. Our simulation results show that the second chaotic attractor serves to "stabilize" the system after voltage collapse. This can be viewed as an advantage of chaos. The deleterious effects of chaos are that the magnitude of the chaotic oscillation may be unacceptable for thermal overload reasons or that its harmonic content could damage generators or other components connected to the network.

Since the period-doubling route to chaos is the outcome of a cascade of bifurcations commencing with the Hopf bifurcation, the prevention of chaos is directly connected to the prevention of the Hopf bifurcation. The Hopf bifurcation was shown to exist because of voltage exciter systems

${ }^{24}$ A subsynchronous resonance signifies an electrical oscillation below the line frequency of $60 \mathrm{~Hz}$. 
in [27]. As shown in Figs. 4-6, the Hopf bifurcation can be prevented by an increase in machine damping. This may also be achieved either by power system stabilizers or by the use of high power solid state switches as described in [20].

\section{ACKNOWLEDGMENT}

The authors are grateful for comments on earlier drafts by Profs. Eyad Abed and Hsiao-Dong Chiang, and Dr. Tom Schneider of EPRI.

\section{REFERENCES}

[1] E. H. Abed and P. P. Variaya, "Nonlinear oscillations in power systems," Int. J. Electric Power and Energy Syst., vol. 6, pp. $37-43,1984$

[2] H. O. Wang, E. H. Abed, and A. M. A. Hamdan, "Bifurcations, chaos and crises in voltage collapse of a model power system," IEEE Trans. Circ. and Syst.-I: Fund. Theory and Applic., vol. 41, pp. 294-302, Apr. 1994

[3] E. H. Abed et al., "Dynamic bifurcations in a power system model exhibiting voltage collapse," Int. J. Bifurcation and Chaos, vol. 3, no. 5, pp. 1169-1176, Oct. 1993.

[4] J. C. Alexander, "Oscillatory solutions of a model system of nonlinear swing equations," Int. J. Electric Power and Energy Syst. vol. 8, no. 3, pp. $130-136,1986$

[5] V. Ajjarapu and B. Lee, "The application of bifurcation theory to study the nonlinear Phenomena in an electrical power system,"IEEE Trans. Power Syst., vol. 7, pp. 424-432, Feb. 1992.

[6] 1992. "Period doubling route to chaos in an electrical power system."IEE Proc. Part C, vol. 104, pp. 490-496, Nov. 1993.

[7] A. Back et al., "DSTOOL: Computer assisted exploration of dynamical systems," Computers and Mathematics, vol. 39, no. 4, pp. 303-309, Apr. 1992.

[8] R. L. Devaney, An Introduction to Chaotic Dynamical Systems. New York: Cummings, 1986.

[9] E. J. Doedel and J. P. Kernévez, "AUTO: Software for continuation and bifurcation problems in ordinary differential equations," Applied Mathematics Rep., Calif. Inst. Tech., 1986.

[10] R. L. Chen and P. P. Varaiya, "Degenerate Hopf bifurcation in power systems," IEEE Trans. Circ. and Syst., vol. 35, pp. 818-824, July 1988.

[11] H. D. Chiang, private communication

[12] H. D. Chiang, M. Hirsch, and F. F. Wu, "Stability regions of nonlinear autonomous dynamical systems," IEEE Trans. Autom. Contr., vol. 33, pp. 16-27, Jan. 1988.

[13] C. De Marco and A. Bergen, "A security measure for random load disturbances in nonlinear power system models," IEEE Trans. Circ. and Syst., vol. CAS-34, pp. 1546-1557, Dec. 1987.

[14] I. Dobson and H. D. Chiang, "Toward a theory of voltage collapse in electric power systems," Syst. Contr. Letts., vol. 13, pp. 253-262, 1989 .

[15] I. Dobson and D. F. Delchamps, "Basin boundaries in the pendulum with nonperiodic forcing," in Proc. 23rd Conf. Inform. Sci. and Syst., Baltimore, MD, 1989.

[16] M. J. Feigenbaum, "Universal behavior in nonlinear systems," Nonlinear Dynamics and Turbulence, G. I. Barenblatt, G. Iooss, and D. D. Joseph, Eds., pp. 101-138, 1983.

[17] C. Grebogi, E. Ott, and J. A. Yorke, "Crises, sudden changes in attractors, and transient chaos," Physica, vol. 7D, pp. 181-200, 1983.

[18] J. Guckenheimer and P. J. Holmes, Nonlinear Oscillations, Dynamical Systems, and Bifurcations of Vector Fields, Applied Mathematical Sciences, vol. 42. Berlin: Springer-Verlag, 1986.

[19] D. Hill and A. Bergen, "Stability analysis of multimachine power networks with linear frequency dependent loads," IEEE Trans. Circ. and Syst., vol. CAS-29, pp. 804-848, Dec. 1982.

[20] N. G. Hingorani, "Flexible AC transmission systems," IEEE Spectrum, vol. 30 , pp. $40-45$, Apr. 1993.

[21] I. Hiskens and D. Hill, "Energy functions, transient stability and voltage behavior in power systems with nonlinear loads," IEEE Trans. Power Syst., vol. 4, pp. 1525-1533, Nov. 1989.
[22] N. Kopell and R. B. Washburn, "Chaotic motions in the twodegree-of-freedom swing equations," IEEE Trans. Circ. and Syst., vol. CAS-29, pp. 738-746, 1982.

[23] H. G. Kwatny, A. K. Pasrija, and L. Y. Bahar, "Static bifurcations in electric power systems: Loss of steady state stability and voltage collapse," IEEE Trans. Circ. and Syst., vol. CAS-33, pp. $981-991,1986$.

[24] E. N. Lorenz, "Deterministic nonperiodic flow," J. Atmospheric Sci., vol. 20, pp. 130-141, 1963.

[25] J. E. Marsden and M. McCracken, The Hopf Bifurcation and Its Applications. New York: Springer-Verlag, 1976.

[26] R. M. May, "Simple mathematical models with very complicated dynamics," Nature, vol. 261, pp. 459-467, 1976.

[27] C. Rajagopalan, P. W. Sauer, and M. A. Pai, "Analysis of voltage control systems exhibiting voltage collapse," in Proc. 28th Conf. on Decision and Contr., Tampa, FL, 1989, pp. 332-335.

[28] D. Ruelle and F. Takens, "On the nature of turbulence," Mathematical Phys., vol. 20, pp. 167-192, 1971.

[29] S. S. Sastry and C. A. Desoer, "Jump behavior of circuits and systems," IEEE Trans. Circ. and Syst., vol. CAS-28, pp. 1109-1124, Dec. 1981.

[30] F. M. A. Salam, J. Marsden, and P. P. Varaiya, "Arnold diffusion in swing equations of a power system," IEEE Trans. Circ. and Syst, vol. 30, pp. 673-688, Aug. 1984.

[31] N. A. Tsolas, A. Araposthatis, and P. P. Varaiya, "A structure preserving energy function for power system transient stability analysis," IEEE Trans. Circ. and Syst., vol. CAS-32, pp. 1041-1049, Oct. 1985

[32] P. P. Varaiya, F. F. Wu, and H. D. Chiang, Bifurcation and Chaos in Power Systems: A Survey, EPRI Rep. TR-100834, Aug. 1992

[33] M. Varghese, H. D. Chiang, and J. S. Thorp, "Computer algorithms in power systems: from constructive methods to truncated fractals," IEEE Trans. Educ., vol. 36, pp. 36-41, Feb. 1993.

[34] S. Wiggins, Introduction to Applied Nonlinear Dynamical Systems and Chaos, Texts in Appl. Mathematics, vol. 2. Berlin: Springer-Verlag, 1990.

[35] J. Zaborsky, G. Huang, B. Zheng, and T. C. Leung, "On the phase portraits of a class of large nonlinear dynamical systems such as the power system," IEEE Trans. Autom. Contr., vol. 33 , pp. $4-15$, Jan. 1988

Chin-Woo Tan received the B.Sc. and Ph.D. degrees in electrical engineering, and the M.A. degree in mathematics, all from the University of California at Berkeley.

From 1991 to 1994 he was a Researcher and Lecturer at the University of California at Berkeley. Since 1994 he has been working at GeoStar Technology in Santa Clara, CA. His research interests are nonlinear dynamics and signal processing.

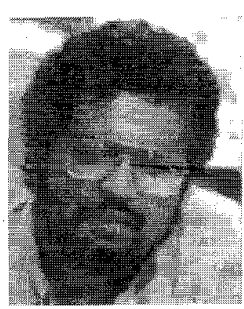

Matthew Varghese received the degrees of B.Tech. (honors), M.S., and Ph.D. degrees in electrical engineering from IIT Kharagpur, Drexel University, and Cornell University, respectively. At Cornell, he was a graduate fellow of the Mathematical Sciences Institute.

$\mathrm{He}$ is currently an Assistant Research Engineer with the Electronics Research Laboratory at the University of California at Berkeley. Prior to that, he was a faculty member at Clarkson University and Harvey Mudd College. 


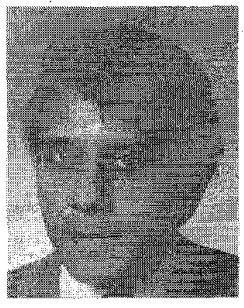

Pravin Varaiya (Fellow, IEEE) is James Fife Professor of Electrical Engineering and Computer Sciences at the University of California at Berkeley, and Director of Califonia PATH, a multiuniversity program of research in Intelligent Transportation Systems. From 1975 to 1992 he also was Professor of Economics at Berkeley. He has taught at MTT and the Federal University of Rio de Janeiro. He was a technical staff member at AT\&T Bell Laboratories during 1962-1963. His research interests are communication networks, transportation systems, and electric power systems.

Dr. Varaiya has held a Guggenheim Fellowship and a Miller Research Professorship.
Felix F. Wu (Fellow, IEEE) is currently Professor and Vice Chairman of the Electrical Engineering and Computer Sciences Department at the University of California at Berkeley. He has held visiting appointments at Eidgenossische Technische Hochschule-Zurich, Shanghai Jiao Tong University, Beijing Tsinghua University, University of Tokyo, and Universita Degli Studi de Cassino. He was a consultant to Pacific Gas and Electric Company, Kansas Gas and Electric, Norwegian State Power Board, Taiwan Power Company, Electric Power Research Institute, and the Chinese Electric Power Research Institute.

Dr. Wu was Chair of Tokyo Electric Power Company's "Frontier Technology for the Future Electric Energy Systems," in 1991. He is now serving as Chairman of the Fellows Working Group of the Power System Engineering Committee. He has served on the Editorial Board of the IEEF Proceedings IEEE Award Board-Prize Paper Committee, and other IEEE, IFAC, and CIGRE committees. 